\title{
Spatial and temporal codes mediate the tactile perception of natural textures
}

\author{
Alison I. Weber ${ }^{a, 1}$, Hannes P. Saal ${ }^{a, 1}$, Justin D. Lieber ${ }^{b}$, Ju-Wen Cheng ${ }^{a, c}$, Louise R. Manfredi ${ }^{a}$, John F. Dammann III ${ }^{a}$ \\ and Sliman J. Bensmaia ${ }^{\mathrm{a}, \mathrm{b}, 2}$ \\ ${ }^{a}$ Department of Organismal Biology and Anatomy and ${ }^{b}$ Committee on Computational Neuroscience, University of Chicago, Chicago, IL 60637; \\ and 'Department of Physical Medicine and Rehabilitation, Chang Gung Memorial Hospital at Linkou, Taoyuan 333, Taiwan
}

Edited* by Ranulfo Romo, Universidad Nacional Autonóma de México, Mexico City, D.F., Mexico, and approved September 10,2013 (received for review March 21, 2013)

\begin{abstract}
When we run our fingers over the surface of an object, we acquire information about its microgeometry and material properties. Texture information is widely believed to be conveyed in spatial patterns of activation evoked across one of three populations of cutaneous mechanoreceptive afferents that innervate the fingertips. Here, we record the responses evoked in individual cutaneous afferents in Rhesus macaques as we scan a diverse set of natural textures across their fingertips using a custom-made rotating drum stimulator. We show that a spatial mechanism can only account for the processing of coarse textures. Information about most natural textures, however, is conveyed through precise temporal spiking patterns in afferent responses, driven by high-frequency skin vibrations elicited during scanning. Furthermore, these texture-specific spiking patterns predictably dilate or contract in time with changes in scanning speed; the systematic effect of speed on neuronal activity suggests that it can be reversed to achieve perceptual constancy across speeds. The proposed temporal coding mechanism involves converting the fine spatial structure of the surface into a temporal spiking pattern, shaped in part by the mechanical properties of the skin, and ascribes an additional function to vibration-sensitive mechanoreceptive afferents. This temporal mechanism complements the spatial one and greatly extends the range of tangible textures. We show that a combination of spatial and temporal mechanisms, mediated by all three populations of afferents, accounts for perceptual judgments of texture.
\end{abstract}

spike timing | roughness | touch | psychophysics | neurophysiology

O ur exquisite tactile sensitivity to surface texture allows us to distinguish silk from satin, or even good silk from cheap silk. However, the neural basis for our ability to identify individual textures has never been investigated. Natural textures can comprise very fine textural features, on the order of micrometers, but also coarser ones on the order of millimeters. Surface features sized over many orders of magnitude must then be fused to yield a unitary percept of texture. At the coarse extreme of this range, Braille dots and gratings have been shown to be encoded in the spatial pattern of activation elicited in slowly adapting type 1 (SA1) afferents (1-4), which densely innervate the primate fingertip. Specifically, the spatial layout of surface features is reflected in the spatial layout of the SA1 response across the sensory sheet, so information about texture can be read out from this neural image, a mechanism that draws an analogy to vision. The most compelling evidence implicating this spatial mechanism in texture perception stems from an elegant series of studies that demonstrate that one of the major perceptual attributes of a textured surface, its roughness, can be predicted from the spatial pattern of activation it elicits in SA1 afferents (1-3). However, most natural textures comprise features that are too fine to be resolved through a spatially modulated neural signal given the limits set by the innervation density (5) and spatial filtering (6) of the skin (Fig. S1). Behavioral results suggest that the tactile perception of fine texture relies on the transduction and processing of complex, high-frequency, and texture-specific vibrations (in the range of $50-800 \mathrm{~Hz}$ ) that propagate over the skin when we scan a surface (7-10). Such skin oscillations would predominantly excite two other populations of afferents, namely rapidly adapting (RA) and Pacinian (PC) fibers (11-13), whose role in texture perception has heretofore never been demonstrated and has, in fact, been called into question (3, 4 ). Not only does this vibration-mediated mechanism of texture perception implicate different afferent populations, it also implies a different coding mechanism for texture, one that relies on temporal rather than spatial structure in afferent responses.

\section{Results}

To investigate how texture is encoded over the range of tangible surfaces, we recorded the activity evoked in SA1, RA, and PC afferents of Rhesus macaques by 55 diverse textured surfaces, delivered to their fingertips using a custom-built rotating drum stimulator. Stimuli ranged from very coarse, such as (Braillelike) embossed dot patterns and corrugated paper (with element sizes on the order of millimeters), to very fine, such as satin and nylon (with elements sized in the tens of micrometers). Many of the textures consisted of elements that span the range of tangible surface elements. To characterize the neural image conveyed by afferents, we computed spatial event plots (SEPs), which are reconstructions of the spatial representation of a stimulus across a population of afferents of a given type (14). Consistent with previous results, the spatial structure of coarse surfaces is faithfully encoded in the spatial pattern of activation in SA1 afferents

\section{Significance}

Our exquisite tactile sensitivity to surface texture allows us to distinguish silk from satin or even good silk from cheap silk. We show that the tactile perception of natural textures relies on two neural mechanisms. Coarse textural features, for example, the individual elements of Braille, are represented in spatial patterns of activation across one population of mechanoreceptive afferents that densely innervate the fingertip skin. In contrast, our ability to discern fine textural features is mediated by the transduction and processing of vibrations produced in the skin during scanning. Indeed, two other populations of vibration-sensitive afferents produce temporally patterned responses to these vibrations, and spiking patterns in these afferent populations convey texture information and shape the way textures are perceived.

Author contributions: A.I.W., J.F.D., and S.J.B. designed research; A.I.W., J.-W.C., L.R.M., and S.J.B. performed research; A.I.W., H.P.S., J.D.L., and S.J.B. analyzed data; A.I.W., H.P.S., J.D.L., and S.J.B. wrote the paper; and J.F.D. set up the experimental apparatus.

The authors declare no conflict of interest.

*This Direct Submission article had a prearranged editor.

Freely available online through the PNAS open access option.

${ }^{1}$ A.I.W. and H.P.S. contributed equally to this work.

${ }^{2}$ To whom correspondence should be addressed. E-mail: sliman@uchicago.edu.

This article contains supporting information online at www.pnas.org/lookup/suppl/doi:10. 1073/pnas.1305509110/-/DCSupplemental. 
(Fig. $1 A$ and $B$ ). However, the responses of these afferents to finely textured surfaces seem to contain little to no meaningful spatial structure.

Spatial Mechanism for Texture Coding. Testing the prevailing theory of texture coding at the somatosensory periphery, we assessed quantitatively the extent to which surface microstructure is reflected in the spatial pattern of activation across SA1 afferents. First, we found that SA1 afferents respond strongly to coarse textures, but only weakly or not at all to finer ones (Fig. $1 C$ ), which casts doubt on their putative role in fine texture processing. Second, although SA1 responses to coarse textures-with spatial periods greater than about $1 \mathrm{~mm}$-are spatially structured, responses to finer textures are no more spatially structured than would be expected by chance (Fig. $1 D$ ). Third, the spatial layout of the SA1 response matches that of the stimulus for coarse but not fine textures (Fig. 1E; SI Materials and Methods), so the structure in the neural image is not congruent with the microstructure of the surface itself. In summary, a spatial mechanism, mediated by SA1 afferents, is viable only for very coarse textures, with elements on the order of millimeters, and fails to account for the coding of texture over a wide range of element sizes. (Note that coarse textures were purposefully overrepresented in the sample used for the spatial analysis.) Although the spatial acuity of the RA signal is

A

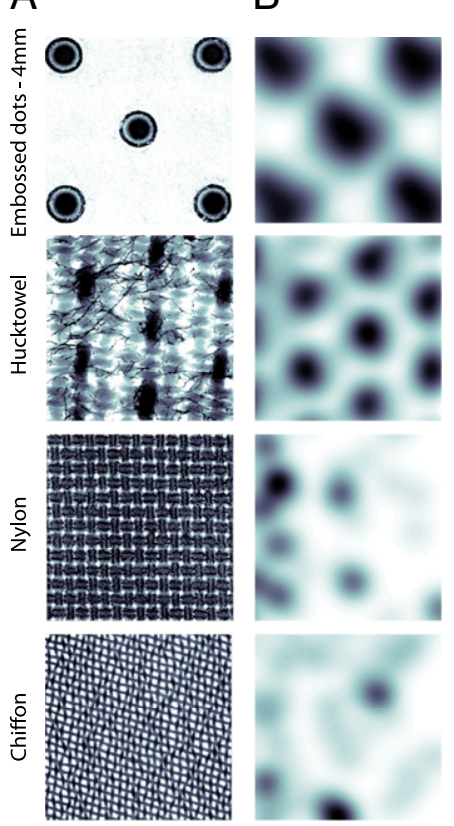

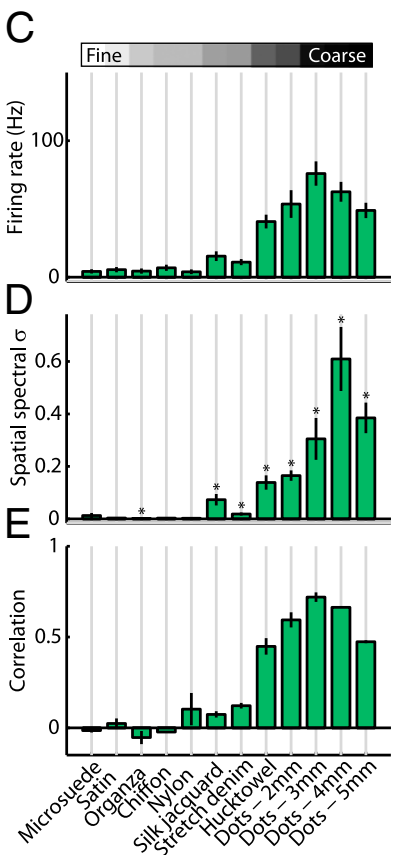

Fig. 1. Spatial hypothesis. (A) Surface microstructure (profilometry) of four texture patches. $(B)$ Spatial pattern of activation (spatial event plots; darker colors indicate stronger neural responses) averaged over all of the SA1 afferents in our sample (spatially aligned across afferents). For coarser textures, such as embossed dots and hucktowel, the spatial structure of the stimulus is well preserved. For finer textures, such as nylon and chiffon, evoked responses are not spatially structured. SEPs are normalized for firing rate to highlight their spatial structure. $(C)$ Average spike rate of SA1 afferents for 12 of the 55 textures that spanned the range from fine to coarse, with textures grouped by roughness from finest to roughest. (As there is no objective measure of coarseness, textures were sorted by perceived roughness.) SA1 afferents produce robust responses only to coarse textures. $(D)$ SD of the power spectra of the SEPs derived from SA1 afferent responses, a measure of spatial patterning. SA1 responses to coarse textures are significantly spatially patterned, whereas those for fine textures are not. Asterisks denote SDs that are significantly different from those expected by chance. $(E)$ Mean correlations between SA1 spatial patterning (SEPS) and surface microstructure. The spatial structure of coarse textures is faithfully reflected in the spatial pattern of activation across SA1 afferents, whereas the structure of fine textures is not lower than is its SA1 counterpart $(15,16)$, RA afferents also densely innervate the fingertip skin (5) and thus might convey textural information spatially. We analyzed the spatial structure of SEPs derived from RA responses using the same approach used for SA1 afferents and found that, as expected, these afferents produce less spatially structured responses than do SA1 afferents (Fig. S2). Finally, PC afferents have very large receptive fields and do not innervate the fingertip densely enough to convey a structured spatial image at the relevant scales.

Temporal Mechanism for Texture Coding. Behavioral studies have suggested that texture-elicited vibrations may play an important role in texture perception $(7-9,17)$. Paired behavioral and neurophysiological studies have shown that the intensity of texture-like skin vibrations is encoded in the strength of the responses evoked in the three populations of mechanoreceptive afferents (12). Furthermore, the frequency composition of the vibrations is encoded in millisecond precision temporal spiking patterns, and this temporal patterning plays an important role in determining how skin vibrations are perceived (13). In light of these previous findings, we considered the possibility that texture information is not encoded in the spatial image, but rather in the time-varying responses of afferents, particularly of RA and PC fibers, as these two afferent populations are highly sensitive to skin vibrations. Examination of the responses of mechanoreceptive afferents to scanned textures shows that the responses are highly repeatable and temporally patterned (Fig. 2). We therefore sought to determine whether these could mediate texture perception. First, we found that, although SA1 afferents respond only weakly $(<10 \mathrm{~Hz})$ to most textures, RA and $\mathrm{PC}$ fibers respond robustly to most, if not all, of them (Fig. S2A; Fig. $3 A$ ). Second, RA and $\mathrm{PC}$ responses to textures are significantly more temporally structured than would be expected by chance (Fig. S3A; Fig. 3B). Third, the frequency composition of afferent responses reflects that of the oscillations elicited in the skin during texture scanning (measured using a laser Doppler vibrometer, Fig. S3B, Fig. 3C; SI Materials and Methods). In fact, afferent responses are more closely associated with the oscillations elicited in the skin than they are with the surface microstructure itself (measured using a laser microscope; Fig. S4; SI Materials and Methods). Indeed, the skin enhances some frequency components and not others $(18,19)$, which highlights the importance of measuring the skin response in studying texture perception. Although PC responses mirrored texture-elicited vibrations across the full range of textures, the match between skin vibrations and RA responses was stronger for coarser textures (Fig. $3 D$ and $E$ ). Fourth, spike timing conveys sufficient information to identify individual textures and does so with high temporal precision, with optimal resolutions of $4 \mathrm{~ms}$ for RA fibers and $2 \mathrm{~ms}$ for PC fibers (Fig. 4A) (13). Indeed, textures can be classified accurately based on the responses of small populations of RA or PC afferents at their optimal resolutions $(83 \%$ with 7 PC fibers; Fig. 4B), suggesting that the whole afferent population (on the order of tens or hundreds of activated units) conveys sufficient information to mediate texture identification. Moreover, although RA and PC afferents convey texture information across the range of element sizes, SA1 responses to all but the roughest textures are uninformative (Fig. $4 B$; Fig. S5). In summary, high-precision temporal spiking patterns, particularly in RA and PC fibers, follow the oscillations produced in the skin during texture scanning and convey sufficient information to mediate texture perception.

Effect of Scanning Speed on Texture-Specific Temporal Spiking Patterns. For nerve fibers to convey unambiguous information about texture, some aspect of their responses must be preserved across scanning speeds. Indeed, our ability to discriminate different textures is mostly independent of speed (20), whereas afferent responses to texture are not. We found that interspike intervals of afferent responses dilate or contract multiplicatively according to the speed ratio (Fig. $4 C$; Fig. S6), and spike trains are considerably 


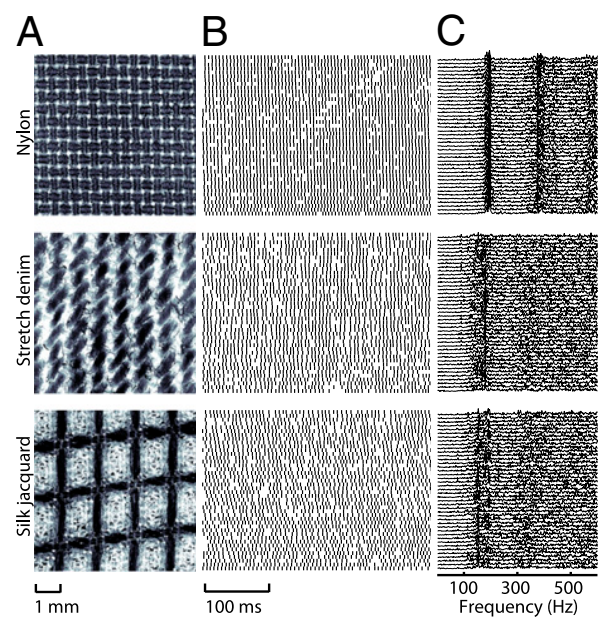

Fig. 2. Responses of one $P C$ afferent to repeated presentations of three textures. $(A)$ Surface microstructure of the three textures. $(B)$ Spike trains elicited over 42 presentations of each texture, with the texture patch progressively displaced along the axis orthogonal to the scanning direction. $(C)$ Power spectrum of the neural response elicited on each trial. Afferent responses to these textures are highly repeatable and temporally patterned along the scanning direction, but there is little to no discernible spatial structure along the orthogonal axis.

more similar to each other when warped by the speed ratio than when warped by other factors $[t$ tests with Bonferroni correction, $t(21)=4.2, \mathrm{t}(19)=5.8$, and $\mathrm{t}(9)=4.5$ for SA1, RA, and PC fibers, respectively, $\mathrm{P}<0.05]$. For example, a spike train evoked by a given texture scanned at $80 \mathrm{~mm} / \mathrm{s}$ is most similar to a spike train evoked by that same texture at $40 \mathrm{~mm} / \mathrm{s}$ when the former is dilated by a factor of 2 . Note that, at this warping factor, the two spike trains are recorded over the same spatial extent of the texture. In fact, the temporal structure is sufficiently conserved that textures can be reliably classified across speeds when responses are appropriately time-warped. Indeed, textures were classified with $89 \%$ accuracy based on the responses of small afferent populations when spike trains obtained at $80 \mathrm{~mm} / \mathrm{s}$ were compared with spike trains obtained at $120 \mathrm{~mm} / \mathrm{s}$ and dilated by a factor of 1.5 (Fig. $4 D$; performance was lower, 62\%, when matching responses obtained at $40 \mathrm{~mm} / \mathrm{s}$, a decrement that is reflected in their perceptual discriminability at this speed). That texture-elicited spike patterns scale with scanning speed is surprising given that they do not simply reflect the spatial structure of the stimulus, but rather the skin response to the stimulus (10). Given the systematic effect of scanning speed on afferent responses, perceptual invariance can be achieved by integrating temporal spiking patterns with the available cutaneous information about scanning speed (21).

Linking Neural Responses to Perception. An important test of a neural coding hypothesis is the establishment of a relationship between the neural response and perception (22). Having shown that temporal spiking patterns convey information about texture, we examined whether these could account for perceptual judgments of surface texture. The most salient perceptual dimension of texture is roughness (23), which is thought to depend on a spatial mechanism. Specifically, the perceived roughness of gratings and dot patterns is well predicted by the spatial variation in SA1 afferents (1-4). That is, to the extent that the response to such surfaces is homogenous over the activated SA1 population, the surface will be perceived as smooth; to the extent that the response is spatially inhomogeneous, the surface will be perceived as rough. Testing the spatial hypothesis with our diverse set of surfaces, we found that this coding mechanism does not generalize to more finely textured surfaces. Spatial variation in SA1 responses is a relatively poor predictor of perceived roughness
$\left(R^{2}=0.64\right)$, particularly for fine textures $\left(R^{2}=0.35\right.$ if dot patterns and gratings are excluded); indeed, textures that yield comparable spatial variation differ up to eightfold in perceived roughness (Fig. $5 A$ ). In contrast, the temporal variation in spiking responses (2), i.e., the degree to which the responses of individual afferents are modulated in time, provides a better fit to the roughness judgments, both for RA $\left(R^{2}=0.88\right.$; Fig. $\left.5 B\right)$ and PC afferents $\left(R^{2}=\right.$ 0.76; Fig. $5 C$ ). Importantly, the predictions of the temporal mechanism are still high when the coarsest textures are excluded $\left(R^{2}=0.77\right.$ and 0.63 for RA and PC afferents, respectively). In fact, spatial variation in SA1 afferents and temporal variation in RA and PC afferents all contribute significantly to perceived roughness across the range of textures tested [overall $R^{2}=$ 0.95; $\Delta R^{2} F$-test: $P<0.001$ for all three predictors, $F(1,50)=$ 13.7, 63.3, and 45.6, with standardized regression coefficients of $0.24,0.45$, and 0.39 , for SA1 spatial variation, RA temporal variation, and PC temporal variation, respectively; Fig. 5D]. In summary, texture perception relies on both a spatial mechanism (dominant for coarse textures) and a temporal mechanism (dominant for fine textures) and is mediated by all three afferent classes.

\section{Discussion}

Two mechanisms underlie the representation of texture in the peripheral nerve: a spatial one and a temporal one, which, in combination, afford us a wide range of tangible textures. Indeed, as previously shown (1-4), larger textural features (on the order of millimeters) are encoded spatially in the responses of SA1 afferents. Here, we show for the first time that finer features are encoded temporally in the responses of RA and PC afferents. Textures that exclusively consist of coarse or fine features will rely on only one of these mechanisms, but textures featuring a range of element sizes will require both. Of the two codes, the temporal one is clearly dominant for our set of natural textures. As a point of reference, if dot patterns and gratings are excluded,
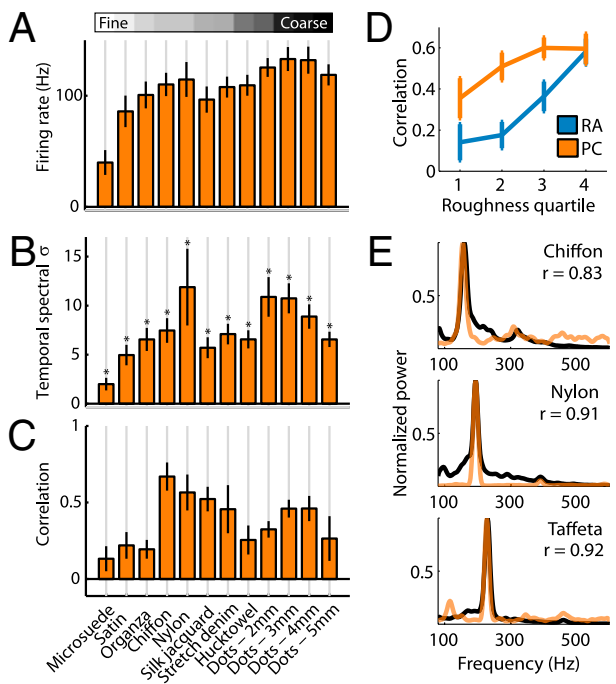

Fig. 3. Temporal hypothesis. (A) Average spike rate of $P C$ afferents evoked by 12 of the 55 textures (same textures as in Fig. $1 C-E$ ). PC afferents respond robustly to all textures. (B) SD of the power spectrum derived from PC spike trains. PC responses are significantly temporally patterned for all 12 textures. (C) Mean correlations between the power spectra of skin vibrations, measured using a laser Doppler vibrometer, and those of the responses of individual afferent PC fibers. The temporal structure of PC responses matches that of skin vibrations. $(D)$ Average correlations between vibratory spectra and RA (blue) and PC (orange) population response spectra. The temporal structure of $P C$ responses matches that of skin vibrations across the range of textures, whereas the temporal structure of RA responses matches that of skin vibrations predominantly for coarse textures. (E) Examples of power spectral densities for skin vibrations (black) and PC population responses (orange) for three fine textures. 


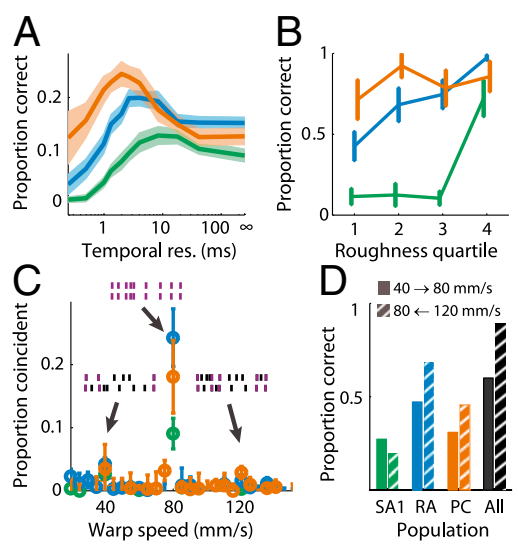

Fig. 4. Discriminating textures based on temporal patterning. $(A)$ Mean classification performance over all 55 textures based on the ISI distributions of the responses of individual SA1 (green), RA (blue), and PC (orange) afferents (chance level is $\sim 1.8 \%$ ). Shaded areas denote the SEM across afferents. Individual PC afferents convey the most texture information and do so at a temporal resolution of $\sim 2$ ms. (B) Population classification performance for textures grouped into quartiles according to their perceived roughness for the three afferent classes at their respective optimal temporal resolutions. Error bars denote the SEM across all textures in each roughness quartile. Although SA1 afferents perform poorly for smooth textures, classification performance based on RA and PC responses is consistently high across the range of tangible textures. (C) Estimate of the proportion of coincident spikes when afferent responses at 40 and $120 \mathrm{~mm} / \mathrm{s}$ are contracted or dilated and aligned to the responses at $80 \mathrm{~mm} / \mathrm{s}$. Error bars denote the SEM across afferents. Insets illustrate a hypothetical spike pattern at three warping factors: The top spike trains are warped according to speed and compared with the bottom spike train, elicited at the reference speed (80 $\mathrm{mm} / \mathrm{s}$ ); coincident spikes are highlighted in purple. When the amount of contraction or dilation corresponds to the ratio of the speeds, afferent responses evoked by a given texture are similar. In other words, increasing the scanning speed preserves the temporal patterning in afferent responses but contracts it temporally in proportion to the speed. $(D)$ Population classification performance across speeds after warping spike trains collected at 40 (solid bars) and 120 (hatched bars) to $80 \mathrm{~mm} / \mathrm{s}$.

SA1 responses convey almost no information about the textures in our set, nor do they account for perceptual judgments of roughness. The temporal mechanism is therefore essential over a wide range of feature sizes. Furthermore, dot patterns and gratings differ from many textures in that they fall at the border between texture and shape, and their spatial layout is perceptually available (and essential in the case of Braille reading). Thus, the spatial image conveyed by SA1 afferents may play a more important role in the perception of shape than it does in the perception of texture, to the extent that these two stimulus properties are distinct. In fact, information about shape is not only conveyed in the spatial response of SA1 (and to some extent) RA afferents, but also in their first spike latencies, suggesting that shape perception may also rely on rate- and timingbased codes $(24,25)$. Note, however, that these two temporal codes-one based on first spike latencies and the other on temporal spiking patterns-likely involve different decoding mechanisms in upstream structures.

The role of RA and PC afferents in texture perception constitutes a deviation from their commonly assigned functional roles. In particular, PC fibers, which were attributed the sole function of mediating the perception of distal events during tool use (26), are now ascribed a second, arguably more important role. Moreover, as texture-induced surface waves travel along the finger onto the palm (18) and elicit measurable vibrations even at the wrist (27), PC afferents with receptive fields centimeters removed from the contact area respond to palpated textures. Thus, texture processing relies not only on fingertip deformations over the contact area (which activate SA1 fibers), but also on the transduction of skin vibrations at locations remote from the contact site, which leads to a considerable amplification of the texture signal (18).

The somatosensory system is typically considered to be a spatial sense, one that draws strong analogies with the visual system (28-30). The extraction of information from oscillations of the somatosensory epithelium implies a complementary mode of processing for the primate somatosensory system, one that draws analogies with the auditory system (31) and with the vibrissal system of rodents (32). Indeed, we show that skin oscillations are transduced into patterns of afferent spiking that reflect the frequency composition of these oscillations. Furthermore, despite the fact that these temporal patterns scale in time with changes in speed, the resulting percept is robust to these changes, a perceptual constancy that may rely on neural mechanisms similar to those that mediate (auditory) timbre constancy (33). That spatial and temporal mechanisms contribute to texture perception raises the question how such disparate representational schemes are integrated in cortex $(34,35)$ to yield a unitary percept.

\section{Materials and Methods}

Stimuli. Textured surfaces were presented to the fingertips of both humans (vibrometry, psychophysics) and anesthetized macaques (neurophysiology) using a custom-built rotating drum stimulator similar to those used in previous studies (36) but larger and more precise. Textured strips $(2.5 \mathrm{~cm}$ wide $\times$ $16 \mathrm{~cm}$ in scanning direction) were wrapped around an acrylic drum $(25.4 \mathrm{~cm}$ in diameter and $30.5 \mathrm{~cm}$ in length). In total, 55 different textures were presented, including gratings (height: $0.74 \mathrm{~mm}$ ) and tetragonal arrays of embossed dots (height: $0.74 \mathrm{~mm}$, diameter: $0.5 \mathrm{~mm}$ ) created from a photosensitive polymer (Printight, Toyobo Co.), as well as finer, more naturalistic textures such as fabrics and sandpapers. Textures were presented with a force of $50 \pm 10 \times g$ and a speed of $80 \pm 0.1 \mathrm{~mm} / \mathrm{s}$. To examine the effects of scanning speed on the neural responses, we also collected data at 40 and $120 \mathrm{~mm} / \mathrm{s}$. On each trial, the drum began to rotate and was lowered onto the fingertip until the desired force was achieved. We only consider
A
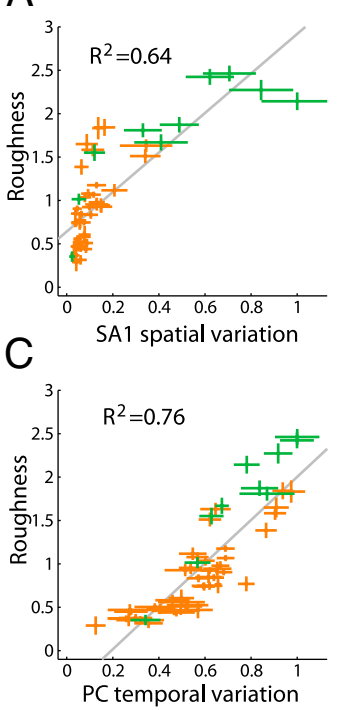
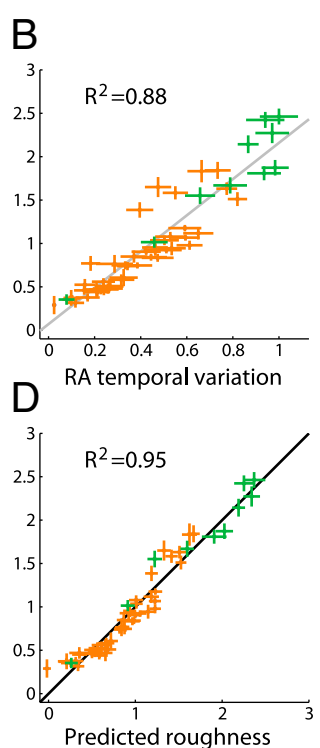

Fig. 5. Linking neural responses to perception. (A) SA1 spatial variation plotted against perceived roughness ratings. Green markers denote embossed dot patterns and gratings and orange markers the remaining textures. Error bars denote SEMs across subjects and neurons along the $y$ and $x$ axes, respectively. The line denotes the line of best fit. SA1 spatial variation is a poor predictor of roughness. ( $B$ and $C$ ) Temporal variation for RA and PC afferents, respectively, plotted against perceived roughness. $(D)$ Combined SA1 spatial variation, and RA and PC temporal variation plotted against perceived roughness. All three predictors contribute significantly to perceived roughness. SEs of the predictors were computed using bootstrapping (Materials and Methods). The solid line indicates unity. Predicted roughness values match the observed ones almost perfectly. 
neuronal responses during the steady-state period when both desired speed and force were achieved. Textures were presented for $2.4,1.2$, or $0.8 \mathrm{~s}$ at 40 , 80 , and $120 \mathrm{~mm} / \mathrm{s}$, respectively, and the interstimulus interval was $3.5 \mathrm{~s}$ to prevent afferent adaptation $(37,38)$. A subset of 12 textures was presented in the SEP protocol, described below.

Psychophysics. Eight subjects (6 males and 2 females; ages 18-31 y) provided informed consent and participated in this study. Subjects sat with the left arm supinated and resting on a support under the drum. Stimuli were presented to the left index fingerpad of each subject. On each trial, the subject was presented with 1 of 55 textures and produced a rating in proportion to its perceived roughness, where a rating of zero denoted a perfectly smooth surface. Each texture was presented once in each of six experimental blocks; ratings were normalized by the mean of each block and averaged, first within and then across subjects. Ratings of roughness were highly consistent across subjects (intersubject correlation: $0.91 \pm 0.03$, mean \pm SD). In this study, roughness judgments were used as a proxy for coarseness, for which there is no objective measure. Indeed, although profilometry certainly provides information about the spatial layout of a texture, it is difficult to translate 3D structure into a measure of coarseness: the material of which a surface is made will play a critical role in determining how its textural elements interact with the skin. All procedures were approved by the Institutional Review Board of the University of Chicago.

Neurophysiology. Extracellular single-unit recordings were collected from the median and ulnar nerves innervating the distal fingertips of six Rhesus macaques (Macaca mulatta) using established procedures $(11,12)$. Anesthesia was maintained using isoflurane, whose effect on mechanoreceptive responses we have shown to be equivalent to that of pentobarbital (39), the anesthetic agent that has been used in most if not all previous studies investigating mechanoreceptive afferents of primates. Data were collected from 15 SA 1 fibers, 13 RA fibers, and 7 PC fibers. Units were classified as SA1, RA, or PC using standard methods (12). Each texture was presented at least twice at each scanning speed. All procedures complied with the National Institutes of Health Guide for the Care and Use of Laboratory Animals and were approved by the Animal Care and Use Committee of the University of Chicago.

Spatial Event Plots. Data amenable to SEP analysis were obtained for only a subset of 12 textures because this experimental protocol is very time consuming. Data for SEPs were obtained by repeatedly passing the stimulus over the receptive field of a single neuron, at $80 \mathrm{~mm} / \mathrm{s}$, with a shift of $500 \mu \mathrm{m}$ along the axis of rotation between each presentation (14). In this way, a different band of the same texture passes over the receptive field with each successive presentation. Because afferent responses are relatively similar across afferents of a given type, spike trains elicited in a single mechanoreceptive afferent by different portions of the stimulus can be used to estimate the spatial pattern of activation elicited by the stimulus across the afferent population (14). Each texture was presented twice at 21 different locations along the axis of rotation, creating an SEP that was $1 \mathrm{~cm}$ wide. The SEPs shown in Fig. $1 B$ were created by first aligning and then averaging the SEPs from all SA1 afferents. SEPs were aligned by finding the $8 \times 8-\mathrm{mm}$ section with the maximum correlation.

SD of the Power Spectrum. With this analysis, we sought to gauge the extent to which afferent responses exhibit periodic structure. In other words, is the neural response more periodic than would be expected by chance? All of the textures tested in this analysis were themselves periodic, so, to the extent that the neuronal response reflected the spatial structure of the stimulus, the response would exhibit periodic structure. We tested whether the spatial pattern of activation was periodic by performing the analysis on the power spectra of SEPs and whether the temporal pattern of activation was periodic by performing the analysis on power spectra of individual spike trains. Both analyses were carried out on the same data, namely the responses used to compute the SEPs: each row corresponds to the response to one scan across the texture, with different rows corresponding to responses evoked by spatially displaced scans.

Spatial analysis. First, we computed the 2D power spectrum of each $10 \times$ $10-\mathrm{mm}$ SEP (binned in $0.5 \times 0.5-\mathrm{mm}$ bins) and averaged the power spectrum across trials for each texture/afferent pair. Second, we collapsed the spectra across the scanning direction to isolate structure that could only be represented spatially (as opposed to temporally), namely structure along the axis perpendicular to the scanning direction. We then computed the SD of each resulting power spectrum. To compute the reliability of the SD, we performed a bootstrapping analysis. On each of 500 iterations, we shuffled the rows to eliminate the spatial structure in the response and recomputed the SD based on the shuffled SEP using the approach described above. The resulting distribution of SDs constituted a null distribution (of SDs obtained in the absence of spatial structure), to which we could compare measured values to gauge their statistical reliability.

Temporal analysis. First, we computed the 1D power spectrum (along the scanning direction) of each spike train used to generate the SEPs and then computed the mean across presentations for each texture/afferent pair (treating each row as an independent and equivalent observation). For this analysis, we use 1.25-ms (0.1-mm) bins. Second, we computed the SD of each resulting mean spectrum. In the bootstrapping analysis, we shuffled the (binned) spike times of the responses evoked in each repetition (500 times) and recomputed the SD based on the shuffled spike trains using the approach described above. The resulting distribution of SDs constituted a null distribution (of SDs obtained in the absence of temporal structure), to which we could compare measured values to gauge their statistical reliability.

Texture Classification from Neural Data. With these analyses, we wished to determine the extent to which textures can be distinguished based on the spiking behavior of afferent populations. To this end, we divided the neural responses recorded during the steady-state period into two consecutive time windows of $500 \mathrm{~ms}$. The underlying assumption was that afferent responses to a given texture should be consistent across time. We then calculated the distance (dissimilarity) between the response evoked by each texture during the first time window and the response evoked by every texture in the second time window (measures of dissimilarity are described below). After calculating pairwise distances between the responses evoked by different textures, we identified which of the 55 textures resulted in the minimum distance. If the response to one texture, measured in the first window, was nearest to the response to the same texture, measured in the second window, the classification was correct; otherwise, the algorithm misclassified the texture. We used two different measures of neural response distance. First, we compared the interspike interval (ISI) distributions (using a variable bin size) of two spike trains by computing the cost of transforming one into the other. ISIs, spanning the range from 0 to $250 \mathrm{~ms}$, were placed into a varying number of bins, from 1 to 512 bins, to vary the temporal resolution from rate to a resolution of $\sim 0.5 \mathrm{~ms}$ by recursively splitting bins in half. Distances between pairs of ISI histograms were then determined by calculating the minimum cost to transform one histogram into another, by adding, deleting, and moving spikes between bins (at unit cost for each operation). Second, we used a spike train distance metric $\left(D_{\text {spike }}\right)$ that calculates the dissimilarity between two spike trains by computing the cost it takes to transform one spike train into another, with a cost of 1 for adding or deleting a spike and a variable cost per unit time for shifting a spike (40). This variable cost determines the temporal resolution of the analysis: ranging from submillisecond to spike count. Spike trains were aligned by computing distances at a variety of different offsets and then choosing the minimum distance to maximally align the phase of the two responses. Although $D_{\text {spike }}$ is computed based on the precise timing of individual spikes, the ISI-based method works by using general statistics of spike timing over a given time window. We found that both methods agreed well in overall classification performance and yielded the same optimal temporal resolution, at which the best performance is achieved (see Fig. $4 A$ for results from the ISI-based classification and Fig. 57 for those based on $D_{\text {spike }}$ ). To classify textures based on the population response, we summed $D_{\text {spike }}$ across afferents at their optimal temporal resolution for each texture pair before finding the texture that yielded the minimum distance (13).

Scaling of Spike Trains According to Scanning Speed. To what extent is the temporal patterning in afferent responses preserved across different scanning speeds? To address this question, we scaled the ISIs of spike trains collected at 40 and $120 \mathrm{~mm} / \mathrm{s}$ to a number of different speeds $v_{i}$ (ranging from 30 to $150 \mathrm{~mm} / \mathrm{s}$ ) by multiplying each ISI by the ratio of the actual speed $v_{m}$ ( 40 or $120 \mathrm{~mm} / \mathrm{s}$ ) to $v_{i}$. For example, we quadrupled ISIs obtained at $120 \mathrm{~mm} / \mathrm{s}$ to generate a hypothetical spike train evoked at $30 \mathrm{~mm} / \mathrm{s}$. We then compared these time-warped spike trains with those collected at the reference speed, $v_{r}(80 \mathrm{~mm} / \mathrm{s})$, by counting the maximum number of coincident spikes (using 4-ms-wide bins) across temporal offsets (to eliminate any discrepancy due to absolute phase, which was not necessarily consistent across speeds; that is, different albeit overlapping extents of the texture were presented at different speeds). To correct for any speed-dependent biases and to focus on fine temporal patterns (on the order of milliseconds) rather than coarse ones, we counted the number of coincident spikes for artificial spike trains, generated from the original ones by scrambling ISIs within 200-ms-long time windows. (One artificial spike train was generated for each measured spike train.) Counts obtained from scrambled ISIs provided an estimate of the number of coincident spikes expected by chance given the coarse temporal 
structure of the response. We then divided the number of coincident spikes above chance as found in the warping analysis by the number of coincident spikes above chance when comparing spike trains from single trials collected at the reference speed of $80 \mathrm{~mm} / \mathrm{s}$. If spike trains collected at different speeds are time-warped versions of each other, our measure exhibits a peak at the reference speed. If, however, spike patterns do not change consistently with scanning speed, but are constant regardless of speed, peaks at 40 and $120 \mathrm{~mm} / \mathrm{s}$ should be observed. Classification across speeds was performed by first warping spike trains collected at 40 and 120 to $80 \mathrm{~mm} / \mathrm{s}$ and then computing $D_{\text {spike }}$ between the original spike trains collected at $80 \mathrm{~mm} / \mathrm{s}$ and the warped ones.

Spatial Variation. To measure the spatial variation in SA fibers, we replicated the exact methods of Connor and Johnson (2). Specifically, SEPs were created by counting the number of whole and fractional ISIs within each $0.5-\mathrm{mm}$ bin. Spatial variation was estimated by convolving a 2D Gabor filter $f$ with each SEP:

$$
f(x, y)=\sin \left\{\frac{2 \pi[x \cdot \sin (\theta)-y \cdot \cos (\theta)]}{\lambda}+\varphi\right\} \cdot \exp \left[\frac{-\left(x^{2}+y^{2}\right)}{2 \sigma^{2}}\right],
$$

where $(x, y)$ is the spatial position, $\theta$ is the orientation of the sinusoidal component of the filter, $\lambda$ is the spatial period of the filter, $\varphi$ is the phase of the sinusoidal component relative to the center of the filter, and $\sigma$ is the SD of the 2D Gaussian component of the filter. Spatial variation was calculated at a range of rotations and translations of the Gabor filter across the field of the SEP and then averaged to obtain the overall spatial variation. We implemented the analysis and optimal parameters used by Connor and Johnson ( $\lambda=2.8 \mathrm{~mm}, \sigma=1.12 \mathrm{~mm}$ ) (2). Because we wished to extend this analysis to all textures, spatial variation was also computed based on reconstructed SEPs. Specifically, the SEP for each texture was generated by sampling rows with replacement from the afferent responses to that texture. The spatial variation was then computed as described above. We verified that these estimated SEPs yielded equivalent spatial variation values by

1. Connor CE, Hsiao SS, Phillips JR, Johnson KO (1990) Tactile roughness: Neural codes that account for psychophysical magnitude estimates. J Neurosci 10(12):3823-3836.

2. Connor CE, Johnson KO (1992) Neural coding of tactile texture: Comparison of spatial and temporal mechanisms for roughness perception. J Neurosci 12(9):3414-3426.

3. Blake DT, Hsiao SS, Johnson KO (1997) Neural coding mechanisms in tactile pattern recognition: The relative contributions of slowly and rapidly adapting mechanoreceptors to perceived roughness. J Neurosci 17(19):7480-7489.

4. Yoshioka T, Gibb B, Dorsch AK, Hsiao SS, Johnson KO (2001) Neural coding mechanisms underlying perceived roughness of finely textured surfaces. J Neurosci 21(17):6905-6916.

5. Johansson RS, Vallbo AB (1979) Tactile sensibility in the human hand: Relative and absolute densities of four types of mechanoreceptive units in glabrous skin. $J$ Physio 286:283-300.

6. Sripati AP, Bensmaia SJ, Johnson KO (2006) A continuum mechanical model of mechanoreceptive afferent responses to indented spatial patterns. I Neurophysiol 95(6):3852-3864.

7. Hollins M, Bensmaïa SJ, Washburn S (2001) Vibrotactile adaptation impairs discrimination of fine, but not coarse, textures. Somatosens Mot Res 18(4):253-262.

8. Bensmala SJ, Hollins M (2003) The vibrations of texture. Somatosens Mot Res 20(1):33-43

9. Bensmaïa S, Hollins M (2005) Pacinian representations of fine surface texture. Percept Psychophys 67(5):842-854.

10. Manfredi LR, et al. (2011) The statistics of natural scenes in tactile texture perception. Soc Neurosci Abstr 37:704.713.

11. Talbot WH, Darian-Smith I, Kornhuber HH, Mountcastle VB (1968) The sense of flutter-vibration: Comparison of the human capacity with response patterns of mechanoreceptive afferents from the monkey hand. I Neurophysio/ 31(2):301-334.

12. Muniak MA, Ray S, Hsiao SS, Dammann JF, Bensmaia SJ (2007) The neural coding of stimulus intensity: Linking the population response of mechanoreceptive afferents with psychophysical behavior. J Neurosci 27(43):11687-11699.

13. Mackevicius EL, Best MD, Saal HP, Bensmaia SJ (2012) Millisecond precision spike timing shapes tactile perception. J Neurosci 32(44):15309-15317.

14. Phillips JR, Johnson KO, Hsiao SS (1988) Spatial pattern representation and transformation in monkey somatosensory cortex. Proc Natl Acad Sci USA 85(4):1317-1321.

15. Phillips JR, Johnson KO (1981) Tactile spatial resolution. II. Neural representation of Bars, edges, and gratings in monkey primary afferents. J Neurophysiol 46(6):1192-1203.

16. Bensmaïa SJ, Craig JC, Yoshioka T, Johnson KO (2006) SA1 and RA afferent responses to static and vibrating gratings. J Neurophysio/ 95(3):1771-1782.

17. Hollins M, Risner SR (2000) Evidence for the duplex theory of tactile texture perception. Percept Psychophys 62(4):695-705.

18. Manfredi LR, et al. (2012) The effect of surface wave propagation on neural responses to vibration in primate glabrous skin. PLOS ONE 7(2):e31203.

19. Scheibert J, Leurent S, Prevost A, Debrégeas G (2009) The role of fingerprints in the coding of tactile information probed with a biomimetic sensor. Science 323(5920):1503-1506.

20. Lederman SJ (1983) Tactual roughness perception: Spatial and temporal determinants. Can J Psychol 37(4):498-511. comparing the spatial variation based on actual and estimated SEPs for the textures that were run in both protocols. For those 12 textures, we found that the correlation between the two quantities was 0.95 , so we used spatial variation based on reconstructed SEPs in the analyses shown in Fig. 5. We also verified that the same conclusions were reached when only the 12 textures run on the SEP protocols were used (Fig. S8).

Temporal Variation. Temporal variation was estimated by convolving a 1D Gabor filter $f$ with the spike trains evoked during individual presentations of each textured surface, binned in 4-ms windows:

$$
f(t)=\sin \left[\frac{2 \pi t}{\lambda}+\varphi\right] \cdot \exp \left[\frac{-t^{2}}{2 \sigma^{2}}\right] .
$$

Filter parameters were optimized for each afferent class individually and were found to be similar for RAs $\left(\lambda=2,857 \mathrm{~ms}, \sigma=14.2 \mathrm{~ms}, \varphi=0.1^{\circ}\right)$ and PCs $\left(\lambda=1,785 \mathrm{~ms}, \sigma=14 \mathrm{~ms}, \varphi=0.3^{\circ}\right)$. Note that large values of $\lambda$ combined with small values of $\varphi$ yield symmetrical filters with one negative component followed by a positive one, the combined width of which is determined by $\sigma$.

Composite Code for Roughness. We calculated a predicted roughness value for each texture using a multiple regression model of the values for each of the three codes. SEs of predicted roughness were computed from bootstrapped values across cells.

ACKNOWLEDGMENTS. We thank Vicky Polashock for providing us with profilometry on the textured stimuli used in this study, Kyler Brown for his contribution to the spatial analysis, and Ed Connor, Richard Williams, Melina Hale, Andrew Pruszynski, and Michael Harvey for helpful comments on a previous version of this manuscript. We also thank our veterinary staff, Craig Wardrip, Marek Niekrasz, Maggie Bruner, Jenny McGrath, and Karin Peterson, for keeping our animals healthy and happy. This work was supported by National Science Foundation Grant IOS-1150209.

21. Dépeault A, Meftah M, Chapman CE (2008) Tactile speed scaling: Contributions of time and space. J Neurophysio/ 99(3):1422-1434.

22. Johnson KO (2000) Neural coding. Neuron 26(3):563-566

23. Hollins M, Bensmaïa S, Karlof K, Young F (2000) Individual differences in perceptual space for tactile textures: Evidence from multidimensional scaling. Percept Psychophys 62(8):1534-1544.

24. Johansson RS, Birznieks I (2004) First spikes in ensembles of human tactile afferents code complex spatial fingertip events. Nat Neurosci 7(2):170-177.

25. Saal HP, Vijayakumar S, Johansson RS (2009) Information about complex fingertip parameters in individual human tactile afferent neurons. J Neurosci 29(25):8022-8031.

26. Johnson KO, Yoshioka T, Vega-Bermudez F (2000) Tactile functions of mechanoreceptive afferents innervating the hand. J Clin Neurophysiol 17(6):539-558.

27. Delhaye B, Hayward V, Lefevre P, Thonnard JL (2012) Texture-induced vibrations in the forearm during tactile exploration. Front Behav Neurosci 6:37.

28. Phillips JR, Johnson KO, Browne HM (1983) A comparison of visual and two modes of tactual letter resolution. Percept Psychophys 34(3):243-249.

29. Bensmaia SJ, Denchev PV, Dammann JF 3rd, Craig JC, Hsiao SS (2008) The representation of stimulus orientation in the early stages of somatosensory processing. $J$ Neurosci 28(3):776-786.

30. Pei YC, Hsiao SS, Craig JC, Bensmaia SJ (2011) Neural mechanisms of tactile motion integration in somatosensory cortex. Neuron 69(3):536-547.

31. Yau JM, Olenczak JB, Dammann JF, Bensmaia SJ (2009) Temporal frequency channels are linked across audition and touch. Curr Biol 19(7):561-566.

32. Arabzadeh E, Zorzin E, Diamond ME (2005) Neuronal encoding of texture in the whisker sensory pathway. PLOS Biol 3(1):e17.

33. Patil K, Pressnitzer D, Shamma S, Elhilali M (2012) Music in our ears: The biological bases of musical timbre perception. PLOS Comput Biol 8(11):e1002759.

34. DiCarlo JJ, Johnson KO, Hsiao SS (1998) Structure of receptive fields in area $3 \mathrm{~b}$ of primary somatosensory cortex in the alert monkey. J Neurosci 18(7):2626-2645.

35. Harvey MA, Saal HP, Dammann JF, 3rd, Bensmaia SJ (2013) Multiplexing stimulus information through rate and temporal codes in primate somatosensory cortex. PLoS Biol 11(5):e1001558.

36. Johnson KO, Phillips JR (1988) A rotating drum stimulator for scanning embossed patterns and textures across the skin. J Neurosci Methods 22(3):221-231.

37. Leung YY, Bensmaïa SJ, Hsiao SS, Johnson KO (2005) Time-course of vibratory adaptation and recovery in cutaneous mechanoreceptive afferents. J Neurophysiol 94(5):3037-3045

38. Bensmaïa SJ, Leung YY, Hsiao SS, Johnson KO (2005) Vibratory adaptation of cutaneous mechanoreceptive afferents. J Neurophysio/ 94(5):3023-3036.

39. Cheng JW, Weber Al, Bensmaia SJ (2013) Comparing the effects of isoflurane and pentobarbital on the responses of cutaneous mechanoreceptive afferents. BMC Anesthesiol 13(1):10.

40. Victor JD, Purpura KP (1997) Metric-space analysis of spike trains: Theory, algorithms and application. Network-Comp Neural 8(2):127-164. 